ultrahigh-quality crystalline silicon, grown at high temperatures, offers comparable or better deep trap densities $\left(10^{8}<n_{\text {traps }}<10^{15} \mathrm{~cm}^{-3}\right)(21,22)$. The exceptionally low trap density found experimentally can be explained with the aid of density functional theory (DFT) calculations performed on $\mathrm{MAPbBr}_{3}$, which predict a high formation energy for deep trap defects when $\mathrm{MAPbBr}_{3}$ is synthesized under Br-rich conditions (e.g., from $\mathrm{PbBr}_{2}$ and MABr), such as is the case in this study (9).

\section{REFERENCES AND NOTES}

1. National Renewable Energy Laboratory, Best ResearchCell Efficiencies; www.nrel.gov/ncpv/images/ efficiency_chart.jpg.

2. A. Kojima, K. Teshima, Y. Shirai, T. Miyasaka, J. Am. Chem. Soc. 131, 6050-6051 (2009).

3. T. C. Sum, N. Mathews, Energy Environ. Sci. 7, 2518-2534 (2014).
4. C. Wehrenfennig, M. Liu, H. J. Snaith, M. B. Johnston, L. M. Herz, Energy Environ. Sci. 7, 2269-2275 (2014)

5. S. D. Stranks et al., Science 342, 341-344 (2013).

6. D. B. Mitzi, Prog. Inorg. Chem. 48, 1-121 (1999).

7. Y. Tidhar et al., J. Am. Chem. Soc. 136, 13249-13256 (2014)

8. M. Xiao et al., Angew. Chem. Int. Ed. 53, 9898-9903 (2014).

9. See supplementary materials on Science Online.

10. J. J. Choi, X. Yang, Z. M. Norman, S. J. L. Billinge, J. S. Owen, Nano Lett. 14, 127-133 (2014).

11. J. R. Haynes, W. Shockley, Phys. Rev. 81, 835-843 (1951).

12. G. Giorgi, K. Yamashita, J. Mater. Chem. A 10.1039/ C4TA05046K (2015).

13. E. Edri, S. Kirmayer, D. Cahen, G. Hodes, J. Phys. Chem. Lett. 4 , 897-902 (2013)

14. M. A. Lampert, P. Mark, Current Injection in Solids (Academic Press, New York, 1970)

15. J. R. Ayres, J. Appl. Phys. 74, 1787-1792 (1993).

16. I. Capan, V. Borjanović, B. Pivac, Sol. Energy Mater. Sol. Cells 91, 931-937 (2007)

17. A. Balcioglu, R. K. Ahrenkiel, F. Hasoon, J. Appl. Phys. 88, 7175-7178 (2000).

18. L. L. Kerr et al., Solid-State Electron. 48, 1579-1586 (2004).
19. C. Goldmann et al., J. Appl. Phys. 99, 034507 (2006).

20. Y. S. Yang et al., Appl. Phys. Lett. 80, 1595-1597 (2002).

21. J. R. Haynes, J. A. Hornbeck, Phys. Rev. 100, 606-615 (1955)

22. J. A. Hornbeck, J. R. Haynes, Phys. Rev. 97, 311-321 (1955)

\section{ACKNOWLEDGMENTS}

We thank N. Kherani, B. Ramautarsingh, A. Flood, and P. O'Brien for the use of the Hall setup. Supported by KAUST (O.M.B.) and by KAUST award KUS-11-009-21, the Ontario Research Fund Research Excellence Program, and the Natural Sciences and Engineering Research Council of Canada (E.H.S.).

\section{SUPPLEMENTARY MATERIALS}

www.sciencemag.org/content/347/6221/519/suppl/DC1

Materials and Methods

Figs. S1 to $\mathrm{S} 12$

References (23-44)

12 November 2014; accepted 19 December 2014

10.1126/science.aaa2725

\title{
High-efficiency solution-processed perovskite solar cells with millimeter-scale grains
}

\author{
Wanyi Nie, ${ }^{1 *}$ Hsinhan Tsai, ${ }^{2 *}$ Reza Asadpour, ${ }^{3} \dagger$ Jean-Christophe Blancon, ${ }^{2} \dagger$ \\ Amanda J. Neukirch, ${ }^{4,5}$ Gautam Gupta, ${ }^{1}$ Jared J. Crochet, ${ }^{2}$ Manish Chhowalla, ${ }^{6}$ \\ Sergei Tretiak, ${ }^{4}$ Muhammad A. Alam, ${ }^{3}$ Hsing-Lin Wang, ${ }^{2} \ddagger$ Aditya D. Mohite ${ }^{1} \ddagger$
}

\begin{abstract}
State-of-the-art photovoltaics use high-purity, large-area, wafer-scale single-crystalline semiconductors grown by sophisticated, high-temperature crystal growth processes. We demonstrate a solution-based hot-casting technique to grow continuous, pinhole-free thin films of organometallic perovskites with millimeter-scale crystalline grains. We fabricated planar solar cells with efficiencies approaching $18 \%$, with little cell-to-cell variability. The devices show hysteresis-free photovoltaic response, which had been a fundamental bottleneck for the stable operation of perovskite devices. Characterization and modeling attribute the improved performance to reduced bulk defects and improved charge carrier mobility in large-grain devices. We anticipate that this technique will lead the field toward synthesis of wafer-scale crystalline perovskites, necessary for the fabrication of high-efficiency solar cells, and will be applicable to several other material systems plagued by polydispersity, defects, and grain boundary recombination in solution-processed thin films.
\end{abstract}

T he recent discovery of organic-inorganic perovskites offers promising routes for the development of low-cost, solar-based clean global energy solutions for the future (1-4). Solution-processed organic-inorganic hybrid

${ }^{1}$ Materials Physics and Application Division, Los Alamos National Laboratory, Los Alamos, NM 87545, USA. ${ }^{2}$ Physical Chemistry and Applied Spectroscopy Division, Los Alamos National Laboratory, Los Alamos, NM 87545, USA. ${ }^{3}$ School of Electrical and Computer Engineering, Purdue University, West Lafayette, IN 47907, USA. ${ }^{4}$ Theoretical Chemistry and Molecular Physics Division, Los Alamos National Laboratory, Los Alamos, NM 87545, USA. ${ }^{5}$ Center for Nonlinear Studies, Los Alamos National Laboratory, Los Alamos, NM 87545 USA. ${ }^{6}$ Materials Science and Engineering, Rutgers University, Piscataway, NJ 08854, USA. *These authors contributed equally to this work. †These authors contributed equally to this work. ‡Corresponding author. E-mail: amohite@lanl.gov (A.D.M.); hwang@lanl.gov (H.-L.W.) perovskite planar solar cells, such as $\mathrm{CH}_{3} \mathrm{NH}_{3} \mathrm{PbX}_{3}$ $(\mathrm{X}=\mathrm{Cl}, \mathrm{Br}, \mathrm{I})$, have achieved high average power conversion efficiency (PCE) values of $\sim 16 \%$ using a titania-based planar structure (1-7) or 10 to $13 \%$ in the phenyl- $\mathrm{C}_{61}$-butyric acid methyl ester (PCBM)based architecture (8-10). Such high PCE values have been attributed to strong light absorption and weakly bound excitons that easily dissociate into free carriers with large diffusion length (11-13). The average efficiency is typically lower by 4 to $10 \%$ relative to the reported most efficient device, indicating persistent challenges of stability and reproducibility. Moreover, hysteresis during device operation, possibly due to defect-assisted trapping, has been identified as a critical roadblock to the commercial viability of perovskite photovoltaic technology (14-17). Therefore, recent efforts in the field have focused on improving film surface cov- erage (18) by increasing the crystal size and improving the crystalline quality of the grains (19), which is expected to reduce the overall bulk defect density and mitigate hysteresis by suppressing charge trapping during solar cell operation. Although approaches such as thermal annealing $(20,21)$, varying of precursor concentrations and carrier solvents (22), and using mixed solvents (23) have been investigated, control over structure, grain size, and degree of crystallinity remain key scientific challenges for the realization of highperformance devices.

Here, we report a solution-based hot-casting technique to achieve $\sim 18 \%$ solar cell efficiency based on millimeter-scale crystalline grains, with relatively small variability ( 2\%) in the overall PCE from one solar cell to another. Figure 1A schematically describes our hot-casting process for deposition of organometallic perovskite thin films. Our approach involves casting a hot $\left(\sim 70^{\circ} \mathrm{C}\right)$ mixture of lead iodide $\left(\mathrm{PbI}_{2}\right)$ and methylamine hydrochloride (MACl) solution onto a substrate maintained at a temperature of up to $180^{\circ} \mathrm{C}$ and subsequently spincoated (15 s) to obtain a uniform film (Fig. 1A). In the conventional scheme, the mixture of $\mathrm{PbI}_{2}$ and $\mathrm{MACl}$ is spin-coated at room temperature and then post-annealed for $20 \mathrm{~min}$ on a hot plate maintained at $100^{\circ} \mathrm{C}$. Figure 1, B to D, illustrates the obtained crystal grain structures using this hotcasting technique for various substrate temperatures and processing solvents. We chose a $\mathrm{PbI}_{2} / \mathrm{MACl}$ molar ratio of 1:1 in all experiments described in this Report to achieve the basic perovskite composition and the best morphology (see fig. S1 for images). We observed large, millimeter-scale crystalline grains with a unique leaf-like pattern radiating from the center of the grain [see scanning electron microscopy (SEM) image of microstructure in fig. S2]. The x-ray diffraction (XRD) pattern shows sharp and strong perovskite (110) and (220) peaks for the hot-casted film, indicating a highly oriented crystal structure (fig. S3). The grain size increases markedly (Fig. 1, $\mathrm{B}$ and $\mathrm{D}$ ) as the substrate temperature increases from room temperature up to $190^{\circ} \mathrm{C}$ or when using solvents with a higher boiling point, such 
A

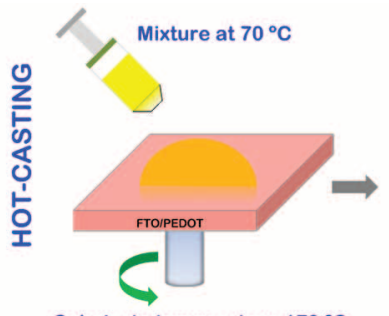

Substrate temperature $170^{\circ} \mathrm{C}$

B

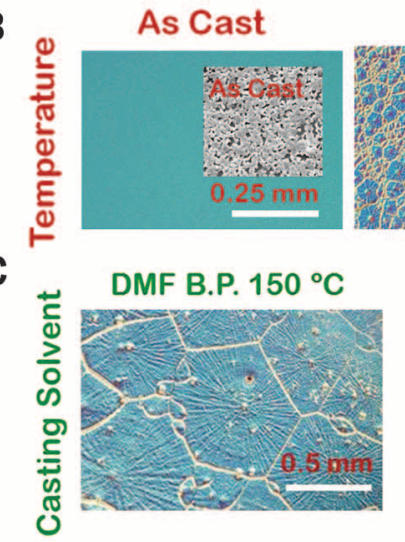

Spin coating or "quenching"

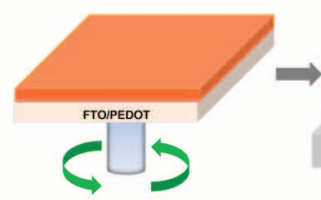

$130^{\circ} \mathrm{C}$

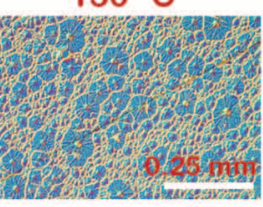

NMP B.P. $202{ }^{\circ} \mathrm{C}$

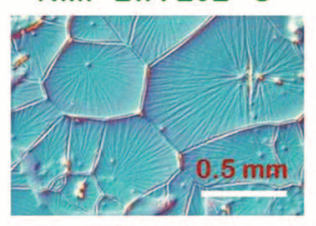

Cooling on Glass

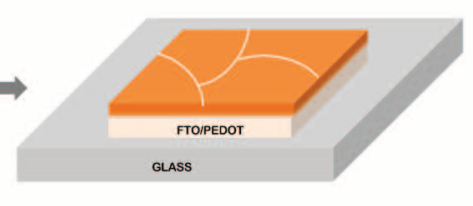

$170^{\circ} \mathrm{C}$

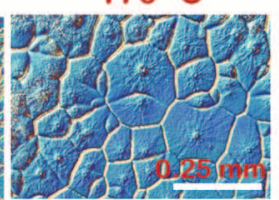

D

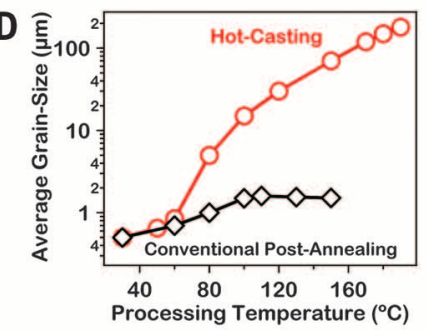

Fig. 1. Processing scheme for perovskite thinfilm using hot-casting methods and observations for large-area millimeter-scale crystal grain formation for a perovskite $\left(\mathrm{PbCH}_{3} \mathrm{NH}_{3} \mathrm{I}_{3-x} \mathrm{Cl}_{x}\right)$ based thin film. (A) Hot-casting scheme for large-area crystal growth [ITO, indium tin oxide; FTO, fluorine-doped tin oxide; PEDOT, poly(3,4ethylenedioxythiophene) polystyrene sulfonate]. (B) Optical micrographs illustrating grain formation as a function of substrate temperature with the casting solution maintained at $70^{\circ} \mathrm{C}$. (C) Largearea grain formation using casting solvents with high boiling points. (D) Comparison of grain size as a function of processing temperature obtained for the hot-casting and conventional post-annealing methods.
A
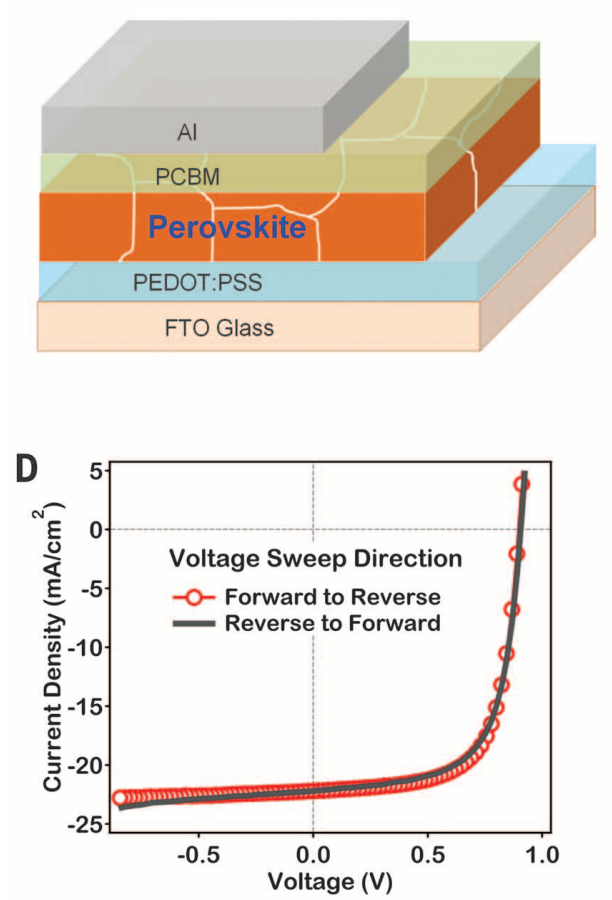
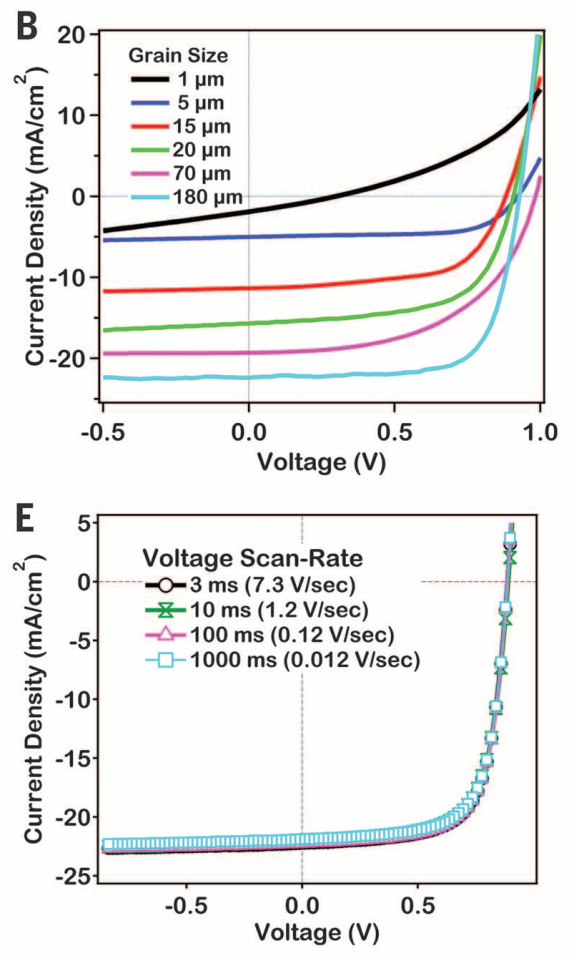
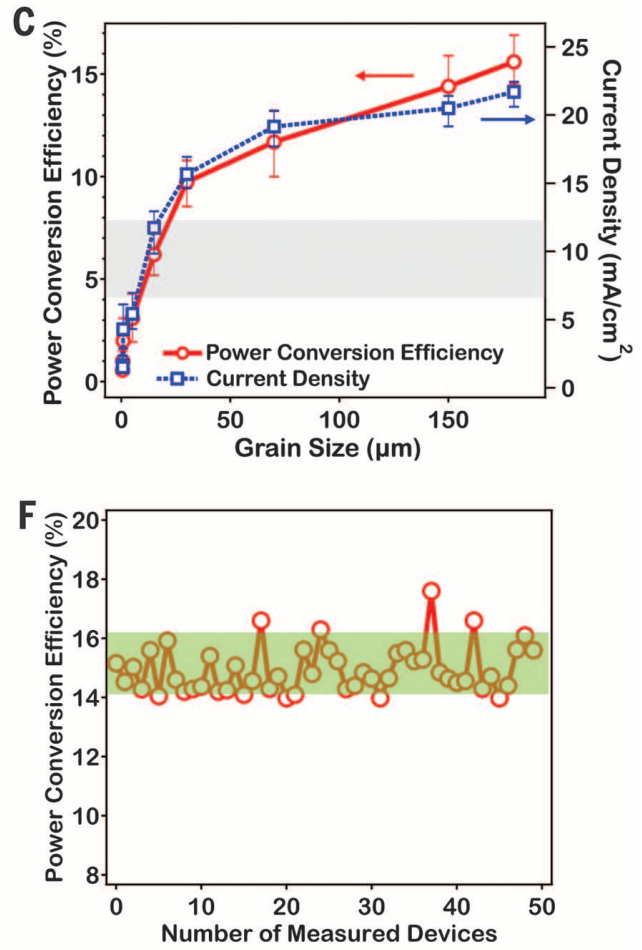

Fig. 2. Solar cell device architecture and performance. (A) Planar device configuration used for this study. (B) J-V curves obtained under AM 1.5 illumination. (C) Average overall PCE (left) and $J_{\text {SC }}$ (right) as a function of crystalline grain size. The average values were obtained by averaging the PCE and $J_{\text {SC }}$ for 25 devices; error bars were calculated by subtracting the average value from max or min values. Gray box represents the range of PCE values obtained using the conventional post-annealing process. (D) Average $J$ - $V$ characteristics resulted by sweeping the voltage from forward to reverse and from reverse to forward bias demonstrates the absence of hysteresis. These curves were obtained by averaging 15 sweeps in each direction. (E) $J$ - $V$ curves at different voltage scan rates in voltage delay time $(\mathrm{ms})$ or $\mathrm{V} / \mathrm{s}$ again demonstrate negligible hysteresis. (F) Variation in PCE for 50 measured devices. 
as $N, N$-dimethylformamide (DMF) or $N$-methyl-2pyrrolidone (NMP) (Fig. 1C).

Our method leads to grain sizes as large as 1 to $2 \mathrm{~mm}$, in comparison with the grain sizes of $\sim 1$ to $2 \mu \mathrm{m}$ typically obtained using the conventional post-annealing process. (See fig. S4 for details of the average grain size calculation procedure.) The major difference between hot-casting and conventional post-annealing is the presence of solvent when heating the substrate. For hot-casting, there is excess solvent present when the substrate is maintained above the crystallization temperature for the formation of the perovskite phase. This allows for the prolonged growth of the perovskite crystals, yielding large crystalline grains. Thus, the use of high-boiling point solvents (see Fig. 1C) provides the ideal conditions for the growth of large crystalline grains, as the excess solvent allows more time for their growth (24) (fig. S5).

Optical images (Fig. 1, B and C) and SEM images (fig. S2) illustrate that the perovskite thin films produced with the hot-casting method (from $100^{\circ} \mathrm{C}$ up to $180^{\circ} \mathrm{C}$ ) are uniform, pinhole-free, and cover the entire substrate. We also used highresolution SEM imaging to evaluate the morphology of the large- and small-area grains and found that except for the size of the grain, there was no observable difference in micromorphology (fig. S2). Preliminary analysis using high-resolution crosssectional SEM imaging coupled with optical polarization microscopy and photoluminescence spectral imaging (figs. S18 to S20) shows consistency of the bulk single-crystalline nature over a large area of the perovskite film. The hot-casting method is applicable for both pure and mixedhalide perovskite combinations (fig. S6) and may lead to the realization of industrially scalable large-area crystalline thin films made from other materials [such as copper zinc tin sulfide (CZTS), copper indium gallium selenide (CIGS), and cadmium telluride (CdTe)] by means of low-temperature, solution-processed, large-area crystal growth.

We fabricated the mixed-halide organometallic perovskite photovoltaic devices using the hotcasting technique in a planar device architecture (Fig. 2A). The current density-voltage $(J-V)$ curves for devices fabricated at various temperatures measured under simulated air mass (AM) 1.5 irradiance of $100 \mathrm{~mW} / \mathrm{cm}^{2}$, calibrated using a NIST-certified monocrystalline Si solar cell (Newport 532 ISO1599), are shown in Fig. 2B. During measurements, a mask was used to confine the illuminated active area to avoid edge effects (25). PCE and shortcircuit current density $\left(J_{\mathrm{SC}}\right)$ are plotted as a function of perovskite grain size in Fig. 2C. An optimal perovskite film thickness of $\sim 450 \mathrm{~nm}$ was fixed for all devices to exclude variations in optical absorption or interference effects due to changes in film thickness. (See fig. S7 and table S1 for details of film thickness optimization and calibration.) We observed a marked increase in $J_{\mathrm{SC}}\left(3.5 \mathrm{~mA} / \mathrm{cm}^{2}\right.$ to $\left.22.4 \mathrm{~mA} / \mathrm{cm}^{2}\right)$, open-circuit voltage $\left(V_{\mathrm{OC}}\right)(0.4 \mathrm{~V}$ to $0.92 \mathrm{~V}$; fig. $\mathrm{S} 8 \mathrm{~A})$, and fill factor $(\mathrm{FF})(0.45$ to 0.82 ; fig. S8B) when the grain size increased from $\sim 1 \mu \mathrm{m}$ up to $\sim 180 \mu \mathrm{m}$, which translated into an increase in the overall PCE from $1 \%$ to $\sim 18 \%$. The $J_{\mathrm{SC}}$ of the devices is in good agreement with the calculated $J_{\mathrm{SC}}$ obtained by integrating the external quantum efficiency (fig. S9). In addition to the high PCE, we found that the solar cell device performance was hysteresisfree, with negligible change in the photocurrent density with either the direction of voltage sweep or the scan rate (or voltage delay time) (Fig. 2, D and E, and fig. S10). The devices exhibited a high degree of reproducibility in the overall PCE (Fig. $2 \mathrm{~F})$. We also applied the hot-casting technique with the conventional mixed-halide precursor ( $\mathrm{PbCl}_{2}$ and MAI, 1:3 molar ratio) but were not able to obtain high-performing devices, most likely because of differences in film morphology and uniformity (fig. S21).

High-performance cells have previously been reported with micrometer-sized grains (1, 26-28). We believe that there are two primary benefits of growing crystals with large grain size: (i) The reduced interfacial area associated with large grains suppresses charge trapping and eliminates hysteresis (irrespective of the direction of voltage sweep or the scan rate), and (ii) larger grains have lower bulk defects and higher mobility, allowing for the photogenerated carriers to propagate through the device without frequent encounters with defects and impurities.

The $J$ - $V$ curves in Fig. 2B support the hypothesis. For example, the reduction in defect-assisted recombination in larger grains is reflected in the observed increase in $V_{\mathrm{OC}}$ from $0.4 \mathrm{~V}$ to $0.94 \mathrm{~V}$ and in $\mathrm{FF}$ from 0.4 to 0.83 with increased grain size. The $J_{\mathrm{SC}}$ value, $22.4 \mathrm{~mA} / \mathrm{cm}^{2}$, is on par with the highest values reported in literature. Indeed, the measured $J_{\mathrm{SC}}, V_{\mathrm{OC}}$, and $\mathrm{FF}$ values for the largegrain device compare favorably with the ShockleyQueisser theoretical limit $\left(J_{\mathrm{SC}} \approx 26.23 \mathrm{~mA} / \mathrm{cm}^{2}\right.$, $V_{\mathrm{OC}} \approx 1.02 \mathrm{~V}$, and $\mathrm{FF} \approx 0.90$ ) for the given device architecture and band alignment (24).

We performed a self-consistent optoelectronic simulation (involving solution of the Maxwell, Poisson, and drift-diffusion equations), which also supports the hypothesis of improved material quality for larger grains (Fig. 3, A to C). On the basis of material parameters from the literature (tables S2 to S5) and bulk defect density and mobility as fitting parameters, the model quantitatively reproduces all the salient features of the $J-V$ characteristic (Fig. 3A) of both $17.7 \%$ (large grain) and $9.1 \%$ (small grain) cells. The key to the efficiency gain is the suppression of defect-assisted recombination in the bulk region: According to the normalized recombination distribution profile across the film thickness (fig. S11B), the majority of recombination for the small-grain device comes from the bulk defect ( $40 \%$ of the loss distributed in the bulk), which suggests that most of the loss comes from defect-assisted recombination. By contrast, for the large-grain device, recombination in the bulk is reduced to $5 \%$ of the overall recombination profile. Moreover, the energy band diagram of the large-grain $17.7 \%$ cell (Fig. 3B) shows that the absorber region is fully depleted, and the built-in electrical field and high mobility allow efficient charge collection. Consistent with the hypothesis, we find that the experimental results can be interpreted only if one assumes that mobility is correlated to grain size (Fig. 3C).

To understand recombination of photogenerated carriers during device operation, we measured
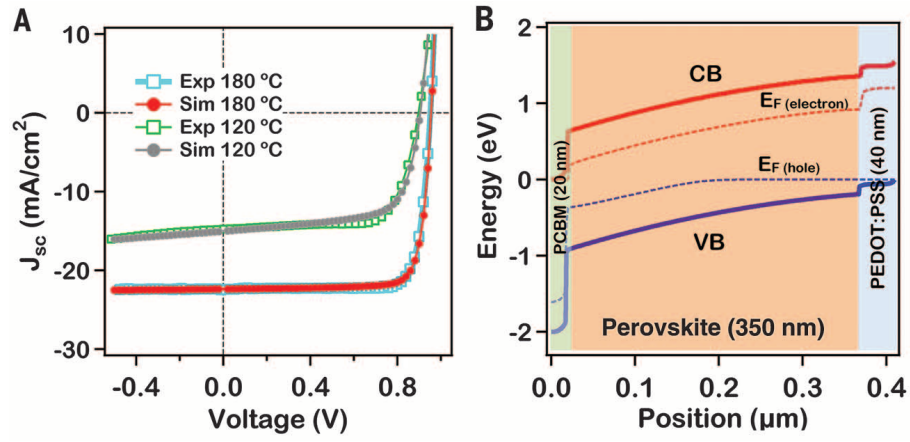

Fig. 3. Self-consistent device simulation attributes the process-dependent PCE gain to the improved mobility of films with larger grains. (A) The simulated $J-V$ characteristics for large- and small-grain devices reproduce the salient features of the corresponding experimental data. (B) Typical energy band diagram at short circuit (SC) indicates the importance of a semi-intrinsic (reduced selfdoping) fully depleted absorber in directing the charge carriers to their respective
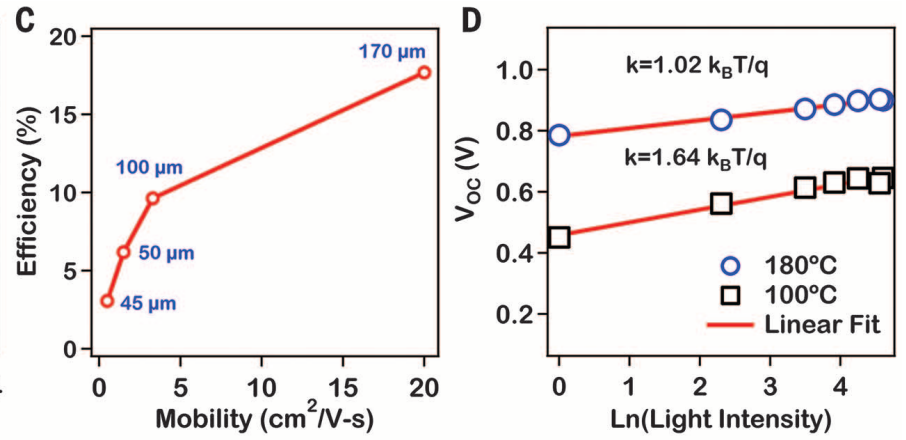

contacts; otherwise, the charge carrier would be lost to recombination in the fieldfree regions. (C) With all other parameters characterized and/or obtained from the literature, the PCE values appear to be correlated to the bulk mobility of the absorber (labels correspond to the average grain size). (D) Experimental data for $V_{\mathrm{OC}}$ as a function of illumination light intensity for a large-grain device $\left(180^{\circ} \mathrm{C}\right)$ and a small-grain device $\left(100^{\circ} \mathrm{C}\right)$ along with the linear fit (red line). 
$V_{\mathrm{OC}}$ as a function of light intensity for large- and small-grain devices (Fig. 3D). By linearly fitting $V_{\mathrm{OC}}$ versus log-scaled light intensity $[\ln (I)]$, we obtained a slope of $\sim 1.0 k_{\mathrm{B}} T / q$ (where $k_{\mathrm{B}}$ is the Boltzmann constant, $T$ is absolute temperature, and $q$ is elementary charge) for a large-grain device (hotcast at $180^{\circ} \mathrm{C}$ ), which suggests that a bimolecular recombination process dominates during device operation (29-31), similar to that observed in highquality semiconductors such as silicon and GaAs. In contrast, for the small-grain device (hot-cast at $100^{\circ} \mathrm{C}$ ), a slope of $1.64 k_{\mathrm{B}} T / q$ is measured, that is an indicator of trap-assisted recombination (Fitting details can be found in (24)).

Finally, direct optical characterization of the material characteristics also supports the hypothesis that material crystalline quality is correlated to grain size. Specifically, we evaluated the overall crystalline quality of large-area grains (24) by performing microphotoluminescence, absorption spectroscopy, and time-resolved photoluminescence measurements on large and small grains. The band-edge emission and absorption for large grains $\left(>10^{3} \mu \mathrm{m}^{2}\right)$ were observed at $1.627 \mathrm{eV}$ and $1.653 \mathrm{eV}$, respectively (Fig. 4A). As the grain size decreased, two concomitant effects were observed: (i) a blue shift of the band-edge photoluminescence by $\sim 25 \mathrm{meV}$ (Fig. 4B), and (ii) linewidth broadening of $\sim 20 \mathrm{meV}$ (Fig. 4C). Such blue shifts were predicted by our density functional theory (DFT) simula- tions (24) (fig. S12) and are possibly attributed to the composition change at the grain boundaries. The increase of emission line width at grain boundaries can be attributed to disorder and defects. We observed a bimolecular recombination process of free electrons and holes for the largegrain crystals by means of time-resolved photoluminescence spectroscopy (Fig. 4D), which is a strong indicator of good crystalline quality (24) (fig. S13). This is in contrast to a monoexponential decay observed in previous reports for small grain size or mesoporous structures $(11,12,32)$ and with our measurements on small grains, and is representative of nonradiative decay due to trap states. These results are also consistent with our earlier measurements of $V_{\mathrm{OC}}$ as a function of light intensity, which suggest reduced trap-assisted recombination in largearea grains.

Beyond our results described above, further enhancements in efficiency can be expected by improving the interface between perovskite and PCBM, obtaining better band alignment, and using an inverted structure. From the perspective of the global photovoltaics community, these results are expected to lead the field toward the reproducible synthesis of wafer-scale crystalline perovskites, which are necessary for the fabrication of high-efficiency single-junction and hybrid (semiconductor and perovskite) planar cells.
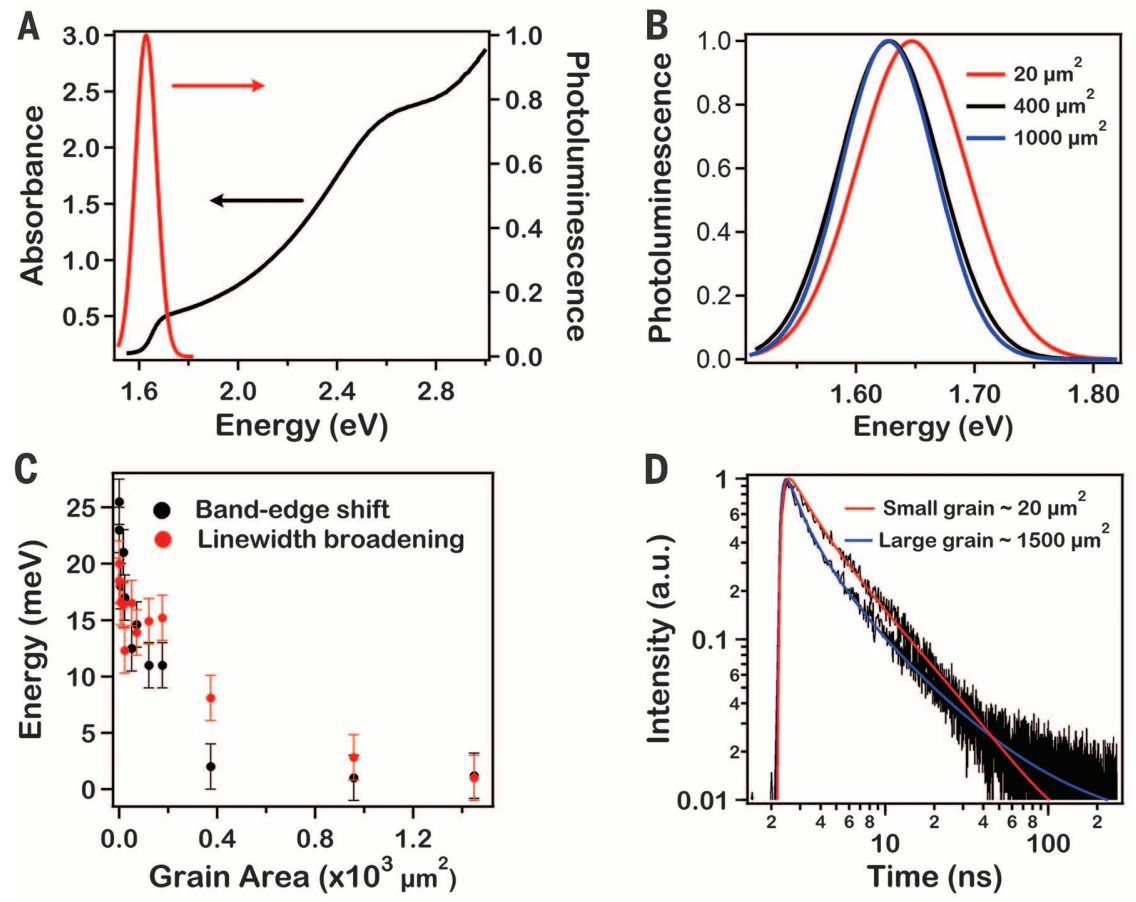

Fig. 4. Spectrally, spatially, and temporally resolved microphotoluminescence spectroscopy. (A) Normalized absorbance (black) and microscopically resolved PL emission spectra (red) obtained for a large-grain sample. (B) Normalized, microscopically resolved emission spectra for different grain sizes. (C) Relative shift and linewidth broadening of the band-edge emission as a function of grain area (with respect to the largest grain). (D) Normalized, microscopically resolved timecorrelated single-photon histograms of both a large and a small grain (black). The red and blue lines are fits to the intensity decay considering interband relaxation, radiative bimolecular recombination, and nonradiative decay into states below the gap (see figs. S13 and S14).

\section{REFERENCES AND NOTES}

1. J. Burschka et al., Nature 499, 316-319 (2013).

2. M. M. Lee, J. Teuscher, T. Miyasaka, T. N. Murakami H. J. Snaith, Science 338, 643-647 (2012).

3. A. Mei et al., Science $345,295-298$ (2014)

4. H. Zhou et al., Science 345, 542-546 (2014)

5. H.-S. Kim et al., Sci. Rep. 2, 591 (2012).

6. M. Liu, M. B. Johnston, H. J. Snaith, Nature 501, 395-398 (2013)

7. N. J. Jeon et al., Nat. Mater. 13, 897-903 (2014).

8. P. Docampo, J. M. Ball, M. Darwich, G. E. Eperon, H. J. Snaith, Nat. Commun. 4, 2761 (2013).

9. O. Malinkiewicz et al., Nat. Photonics 8, 128-132 (2014).

10. J. Seo et al., Energy Environ. Sci. 7, 2642-2646 (2014).

11. S. D. Stranks et al., Science 342, 341-344 (2013).

12. G. Xing et al., Science 342, 344-347 (2013).

13. J. S. Manser, P. V. Kamat, Nat. Photonics 8, 737-743 (2014).

14. M. Grätzel, Nat. Mater. 13, 838-842 (2014).

15. M. D. McGehee, Nat. Mater. 13, 845-846 (2014).

16. R. S. Sanchez et al., J. Phys. Chem. Lett. 5, 2357-2363 (2014).

17. H. J. Snaith et al., J. Phys. Chem. Lett 5, 1511-1515 (2014).

18. G. E. Eperon, V. M. Burlakov, P. Docampo, A. Goriely, H. J. Snaith, Adv. Funct. Mater. 24, 151-157 (2014).

19. M. Xiao et al., Angew. Chem. Int. Ed. 53, 9898-9903 (2014).

20. B. Conings et al., Adv. Mater. 26, 2041-2046 (2014).

21. A. Dualeh et al., Adv. Funct. Mater. 24, 3250-3258 (2014).

22. Q. Wang et al., Energy Environ. Sci. 7, 2359-2365 (2014).

23. H.-B. Kim et al., Nanoscale 6, 6679-6683 (2014).

24. See supplementary materials on Science Online.

25. [Editorial] Nat. Photonics 8, 665 (2014).

26. Q. Chen et al., J. Am. Chem. Soc. 136, 622-625 (2014).

27. G. Grancini et al., J. Phys. Chem. Lett. 5, 3836-3842 (2014).

28. Z. Xiao et al., Adv. Mater. 26, 6503-6509 (2014).

29. S. R. Cowan, A. Roy, A. J. Heeger, Phys. Rev. B 82, 245207 (2010)

30. C. M. Proctor, C. Kim, D. Neher, T.-Q. Nguyen, Adv. Funct. Mater. 23, 3584-3594 (2013).

31. C. M. Proctor, M. Kuik, T.-Q. Nguyen, Prog. Polym. Sci. $\mathbf{3 8 ,}$ 1941-1960 (2013).

32. C. Wehrenfennig et al., Adv. Mater. 26, 1584-1589 (2014).

\section{ACKNOWLEDGMENTS}

Work at Los Alamos National Laboratory (LANL) was supported by the U.S. Department of Energy, Office of Basic Energy Sciences, Work Proposal 08SPCE973 (W.N., G.G., and A.D.M.) and by the LANL LDRD program XW11 (A.D.M., H.-L.W., and S.T.). This work was done in part at the Center for Integrated Nanotechnologies, an Office of Science User Facility. Work at Purdue University was supported by the U.S. Department of Energy under DOE Cooperative Agreement no. DE-EE0004946 ("PVMI Bay Area PV Consortium"). We thank C. Sheehan for the high-resolution cross-sectional SEM images. A.J.N. and S.T. thank C. Katan, J. Even, L. Pedesseau, and M. Kepenekian for useful discussions as well as starting coordinates for bulk perovskites. Author contributions: A.D.M. conceived the idea, designed and supervised experiments, analyzed data, and wrote the manuscript. H.-L.W. and H.T. designed the synthesis chemistry for perovskite thin-film growth and analyzed data. W.N. developed the hot-casting, slow-quenching method for large-area crystal growth along with H.T. and also performed device fabrication and solar cell testing, $x$-ray diffraction and analyzed the data. J.-C.B. performed optical spectroscopy measurements, analyzed the data under the supervision of J.J.C. R.A. performed device modeling simulations. M.A.A. conceived the device modeling, supervised the device modeling, analyzed crystal growth mechanisms, and co-wrote the paper. A.J.N. performed DFT calculations under the guidance of S.T., who designed the DFT calculations, analyzed the data, and provided guidance to the project. G.G. and M.C. conceived the XRD measurements and analyzed the data, co-designed the experiments, and contributed to the organization of the manuscript. All authors have read the manuscript and agree to its contents.

\section{SUPPLEMENTARY MATERIALS}

www.sciencemag.org/content/347/6221/522/suppl/DC1

Materials and Methods

Supplementary Text

Figs. S1 to S21

Tables S1 to S5

References (33-64)

9 October 2014; accepted 23 December 2014 10.1126/science.aaa0472 